BIO Web of Conferences 1, 00101 (2011)

DOI: $10.1051 /$ bioconf $/ 20110100101$

(C) Owned by the authors, published by EDP Sciences, 2011

\title{
Multisensory integration impacts coordination
}

\author{
G. Zelic ${ }^{*}$, D. Mottet*, J. Lagarde* \\ (*) Movement To Health, EuroMov, Montpellier 1 University, 700 Av. Pic Saint Loup 34090 \\ Montpellier, France
}

\begin{abstract}
E-mail: gregory.zelic@univ-montp1.fr,denis.mottet@univ-montp1.fr,julien.lagarde@univmontpl.fr
\end{abstract}

\begin{abstract}
Behavioral benefits have been recently evidenced via multisensory integration process. Reaction times, detection or synchronization performances could be made more efficient by a bimodal stimulation than by its unimodal components. We questioned about the generalization of such results on a more challenging behavioral task. We used a well-known bimanual coordination task in which participants have to synchronize with audio, tactile or audio-tactile metronomes. Results show that according to the intrinsic parameters of the metronome, audio-tactile events could lead to higher coordination performance scores than its corresponding unimodal components.
\end{abstract}

\section{Introduction}

The actual research in behavioral neuroscience domain was focused on how the brain weighs and crosses the informational flows from different sensory modalities, i.e. how it combines and integrates them to product a unified percept. Most of the studies report potential performance profits observed by providing combination of sensorial stimuli compared to unimodal ones [1]. These notably evidenced the role of multisensory information in the detection and localization of external events [2,3], in the variation of the reaction time $[4,5]$, or more recently in the production of timed actions [6]. Such behavioural results have notably contributed to clarify integration properties of cross-modal interactions [7]. Multisensorial integration suppose for example to respect a more or less wide "integration" window, that is to say a certain spatial and temporal concordance between the at least two sensorial cues $[8,9]$.

Nevertheless, these tasks remain confined to motor responses directly related to the provided stimuli: what is measure is the reaction time variability or the synchronisation errors (asynchrony) consequently with provided stimuli.

What appears to be of another behavioural challenge are biological coordination's. These latter are of major interest in human movement research. It necessarily implies the cooperation between at least two components dedicated to a common motor goal. Since about twenty years, synergetic concepts led to the achievement of more and more understanding of coordinated movement pattern as for example interpersonal [10] or interlimb coordination's [11-13].

We study here how multisensory integration process can improve a human coordination

\section{Method}

We asked eight participants to move their index fingers in an antiphase initial coordinative mode (opposite movements of the fingers performed simultaneously). They have to match their movements with several conditions of metronome and to keep their eyes closed during data collection. They were first instructed to try to stay with the metronome and secondly to not intervene if they felt the initial pattern starting to change, but to continue with whatever most comfortable pattern.

We parameterized the metronomes in two ways. First the modality involved: metronomes could be either auditory $(0.08 \mathrm{~s}, 300 \mathrm{~Hz})$ or tactile $(0.08 \mathrm{~s}, 300$ $\mathrm{Hz}$, fixed on the pulp of each auricular) or audio-tactile (superposition of the both).

We tested in addition three structure parameters. In each modality conditions, participants have to perform in a single-metronome condition, in a doublemetronome condition and in an antiphase-metronome one. In the single-metronome condition, metronome frequency began at $1 \mathrm{~Hz}$ and increased in 0.2 steps to $2.8 \mathrm{~Hz}$. Participants were asked to realize one complete cycle for each metronome beat by especially matching the flexion of the right finger with the beat. In the

This is an Open Access article distributed under the terms of the Creative Commons Attribution-Noncommercial License 3.0, which permits unrestricted use, distribution, and reproduction in any noncommercial medium, provided the original work is properly cited. 
antiphase-metronome condition, right and left beat are presented simultaneously so that participants have to match right and left finger flexion with the respective right and left beat. The metronome frequency of a beat corresponds to the frequency scale previously described. Finally in the double metronome condition, metronome frequency went from $2 \mathrm{~Hz}$ to $5.6 \mathrm{~Hz}$ in 0.4 $\mathrm{Hz}$ steps. Here participants were instructed to move so that each metronome beat corresponded with either a finger flexion or extension [14].

Participants performed ten seconds in each of ten plateaus.

\section{Preliminary Results}

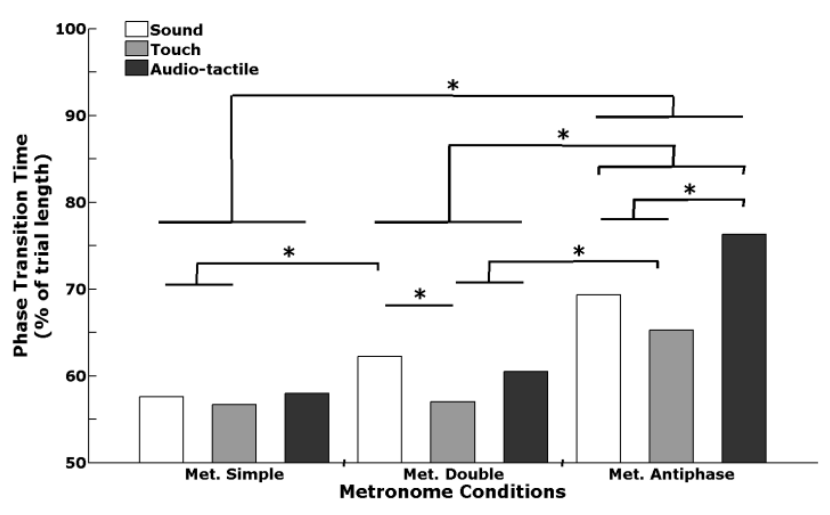

Figure 1: time (\%) during which the initial antiphase coordination mode is maintained

We used an analysis of variance with repeated measures at two factors (two-factor ANOVA), with each one three modalities, to assess the significance of the effects of the different metronomes. Because of the sphericity constraint we report all $\mathrm{p}$ values after Huynh-Feldt correction. We assessed the differences between pair of means with the Fisher's least significant difference (LSD) post hoc-test.

We focused on the time during which participants was able to maintain the initial antiphase coordination mode. The ANOVA applied on this phase transition time indicated an effect of the metronome stucture (Figure $1 ; \mathrm{F}(2,18)=11.92, \mathrm{P}=.0005)$ as of the metronome sensory modality $(\mathrm{F}(2,18)=3.71, \mathrm{P}=$ $.044)$ and an effect of the interaction between the two $(\mathrm{F}(4,36)=3.6, \mathrm{P}=.014)$.

Post-hoc comparisons showed first that the antiphase audio-tactile condition pushed back the phase transition time when compared to others metronome conditions and especially both antiphase unimodal conditions.
Moreover we underline that the audio, tactile and audio-tactile antiphase conditions appears to lead to more higher score of phase transition time than respectively the audio, tactile and audio-tactile modalities in the simple and double metronome conditions.

Finally, we found that the audio double metronome allowed a gain of stability of the initial antiphase coordination mode when compared to the audio and tactile simple metronome and the tactile double one.

\section{Conclusion}

We show that a coordinated pattern of a bimanual rhythmic activity is made more efficient by an audiotactile metronome rather than its unimodal auditory and tactile constituent ones. For the first time, we present here that multisensory integration process can improve a human coordination. We judge this result of first interest especially for two reasons:

First audio-tactile interaction is the least known bimodal interaction. The predominant impact of visual cues in our daily activities has mainly oriented research around this sensory modality. Then little is known about the mutual effects of sound and touch on their respective integrative processes. We reinforce here the idea that the audio-tactile integration process may be the means of improving coordination without overinvesting the visual way.

Secondly we have crossed the recurrent and interdisciplinary question of (multi)sensory integration and our specific knowledge's about biological behaviour and especially coordination. We have brought here the first evidence of a more efficient coordination via added multimodal informations rather than via unimodal ones. Coordination doesn't deal with the speed of the integration processes which is tested in detection tasks for example. Without clearly clarifying the subjacent neuronal process and the responsible brain regions, this result reinforces the hypothesis of motor facilitation thought neuronal interaction and multisensorial neurons networks.

What appears here to be of first interest is that parameters of the metronome matters. The relevance of the matching between the intrinsic antiphase relative phasing between the both fingers and the antiphase metronome clearly highlights the key role of the relative phasing in the effective integration of these audio-tactile cues with an interlimb coordination pattern.

Moreover, this experiment confirms results of Fink [14] about the greater stabilization effect of an audio double-metronome than an audio simple metronome. 
However, the present results post the limits of the hypothesis of an amodal effect of these metronome structure differences. This difference between the simple and the double metronome was not found for the tactile and audio-tactile modalities.

Finally the present results notably offer prospects on the use of added tactile information. They fall under the current debate of the real impact of a tactile stimulus on motor performance between enhanced discrimination and sensorimotor attenuation hypothesis [15], by showing the various impact of such metronome according to their intrinsic structure parameters.

\section{References}

[1] Meredith, M.A. and B.E. Stein. Interactions among converging sensory inputs in the superior colliculus, Science, 221(4608), pages. 389-91, 1983

[2] Stein, B.E., W. Scott Huneycutt, and M. Alex Meredith. Neurons and behavior: the same rules of multisensory integration apply, Brain Research, 448(2), pages. $355-358,1988$

[3] Stein, B.E., et al. Behavioral indices of multisensory integration: orientation to visual cues is affected by auditory stimuli, Journal of Cognitive Neuroscience, 1(1), pages. 12-24, 1989

[4] Hughes, H.C., et al.. Visual-auditory interactions in sensorimotor processing: Saccades versus manual responses, Journal of Experimental Psychology: Human Perception and Performance, 20(1), pages. 131, 1994

[5] Schröger, E. and A. Widmann, Speeded responses to audiovisual signal changes result from bimodal integration. Psychophysiology, 1998. 35(6): p. 755-759.

[6] Wing, A.M., Doumas, M. and Welchman, A.E. Combining multisensory temporal information for movement synchronization, Experimental Brain Research, 200(3), pages. 277-282, 2010

[7] Lagarde, J., Kelso, J.A.S Multimodal coordination dynamics, Experimental Brain Research,173, 673-688, 2006.

[8] Elliott, M.T., Wing, A.M. and Welchman, A.E. Multisensory cues improve sensorimotor synchronization, European Journal of Neuroscience, 31(10), pages. 1828-1835, 2010

[9] Diederich, A. and H. Colonius. Bimodal and trimodal multisensory enhancement: effects of stimulus onset and intensity on reaction time. Attention, Perception, \& Psychophysics, 66(8), pages. 1388-1404, 2004

[10] Varlet, M., et al.. Social postural coordination. Journal of Experimental Psychology: Human Perception and Performance, 37(2): p. 473, 2011
[11] Haken, H., Kelso, J.A. and H. Bunz. A theoretical model of phase transitions in human hand movements, Biol Cybern, 51(5), pages. 347-56, 1985

[12] Kelso, J.A.S., Dynamic patterns: The selforganization of brain and behavior, The MIT Press, 1995

[13] Turvey, M.T. Coordination, Am Psychol, 45(8), pages. 938-53, 1990

[14] Fink, P.W., et al.. Local and global stabilization of coordination by sensory information, Exp Brain Res, 134(1), pages. 9-20, 2000

[15] Voss, M., et al.. Sensorimotor attenuation by central motor command signals in the absence of movement, Nat Neurosci, 9(1), pages. 26-7, 2006 